\title{
Photon or Proton Therapy for Adolescent and Young Adult Tumors Focused on Long-Term Survivors
}

Masashi Mizumoto ${ }^{1}$, Yoshiko Oshiro ${ }^{2}$, Kayoko Tsujino ${ }^{3}$, Shosei Shimizu ${ }^{4}$, Takashi Iizumi ${ }^{4}$, Haruko Numajiri $^{4}$, Kei Nakai ${ }^{1}$, Toshiyuki Okumura ${ }^{4}$, Toshinori Soejima ${ }^{5}$, Hideyuki Sakurai ${ }^{1}$

1. Department of Radiation Oncology, University of Tsukuba Hospital, Tsukuba, JPN 2. Department of Radiation Oncology, Tsukuba Medical Center Hospital, Tsukuba, JPN 3. Department of Radiation Oncology, Hyogo Cancer Center, Akashi, JPN 4. Department of Radiation Oncology, Proton Medical Research Center, University of Tsukuba Hospital, Tsukuba, JPN 5. Department of Radiation Oncology, Kobe Proton Center, Kobe, JPN

Corresponding author: Masashi Mizumoto, mizumoto@pmrc.tsukuba.ac.jp

\section{Abstract}

\section{Background}

This study was conducted to evaluate late toxicities in adolescent and young adult (AYA) patients who received photon or proton therapy.

\section{Methodology}

A total of 106 AYA patients who received proton and photon therapy and were followed-up for more than two years were retrospectively evaluated. The median age of patients was 22 years (range, 15-29 years). A total of 47 patients were male and 59 were female. A total of 35 and 71 patients received photon and proton therapy, respectively. All but one patient received radiotherapy with curative intent. The target disease was benign and malignant in 28 and 78 patients, respectively.

\section{Results}

The median follow-up period in all patients was 62 months (range: 24-293 months). Grade 3 or higher toxicity was observed in 20 patients. There was one case of grade 5 toxicity (myelodysplastic syndrome), which was probably due to chemotherapy. No other secondary cancers were observed. Regarding life events, 15 and 88 patients were married and unmarried at the start of radiotherapy, respectively. Of the 88 unmarried patients, five were married after radiotherapy. Occupation and education were evaluated in 71 patients. Of the 71 patients, 33 were students, 21 were employed, and 16 were unemployed. Of the 33 students, eight were employed and 11 were at a higher educational grade after radiotherapy. Of the 21 employed patients, 17 had the same jobs and four had lost their jobs after radiotherapy. For the 16 unemployed patients, all remained unemployed.

Review began 04/08/2021 Review ended 04/16/2021 Published 04/22/2021

\section{๑) Copyright 2021}

Mizumoto et al. This is an open access article distributed under the terms of the Creative Commons Attribution License CC-BY 4.0., which permits unrestricted use, distribution, and reproduction in any medium, provided the original author and source are credited.

\section{Conclusions}

This study is one of the largest studies to focus on life after radiation therapy among AYAs and suggests that cancer treatment has an influence on life events.

Categories: Pediatrics, Radiation Oncology, Oncology

Keywords: adolescent and young adult, aya, radiotherapy, proton beam therapy

\section{Introduction}

The definition of adolescent and young adults (AYAs) is different among countries [1]. In the United States, AYAs are patients aged between 15 and 39 years at cancer diagnosis, and in the United Kingdom, AYAs include patients aged between 13 and 24 years. However, a clear definition has not yet been established in other countries. For patients in these age groups, there is a gap in oncology services between pediatric and adult patients. Both rare and common malignancies are observed in AYAs. However, malignancies common among adults are not common in AYAs. Therefore, there are fewer studies providing information about malignancies in AYAs. For example, there is no clear consensus on whether AYAs with Hodgkin's lymphoma should be treated with a pediatric or adult trial regimen; therefore, in 2018, the Children's Oncology Group compared clinical trials and suggested that the treatment outcomes for AYAs are better in pediatric trials than adult trials [2].

In addition, late toxicities are a challenge for AYAs in association with improvement of treatment results. However, life stages rapidly change in the AYA age groups, and these patients face different psychosocial, emotional, and physical issues than pediatric and adult patients [3]. Therefore, we consider that adequate support is necessary for each personal situation. 


\section{Cureus}

In this study, we focused on life events, as well as late toxicities, in AYA patients who received photon and proton radiotherapy in two institutions in Japan.

\section{Materials And Methods}

A total of 106 AYA patients who received proton and photon therapy at two institutions in Japan were retrospectively analyzed. Photon and proton therapy were performed at separate institutes. Patients who received radiotherapy, were between 15 and 29 years of age, and were followed up for more than two years were enrolled in this study [4]. This study was approved by the institutional review board (Tsukuba Clinical Research \& Development Organization; R01-144). Patient characteristics are shown in Table 1.

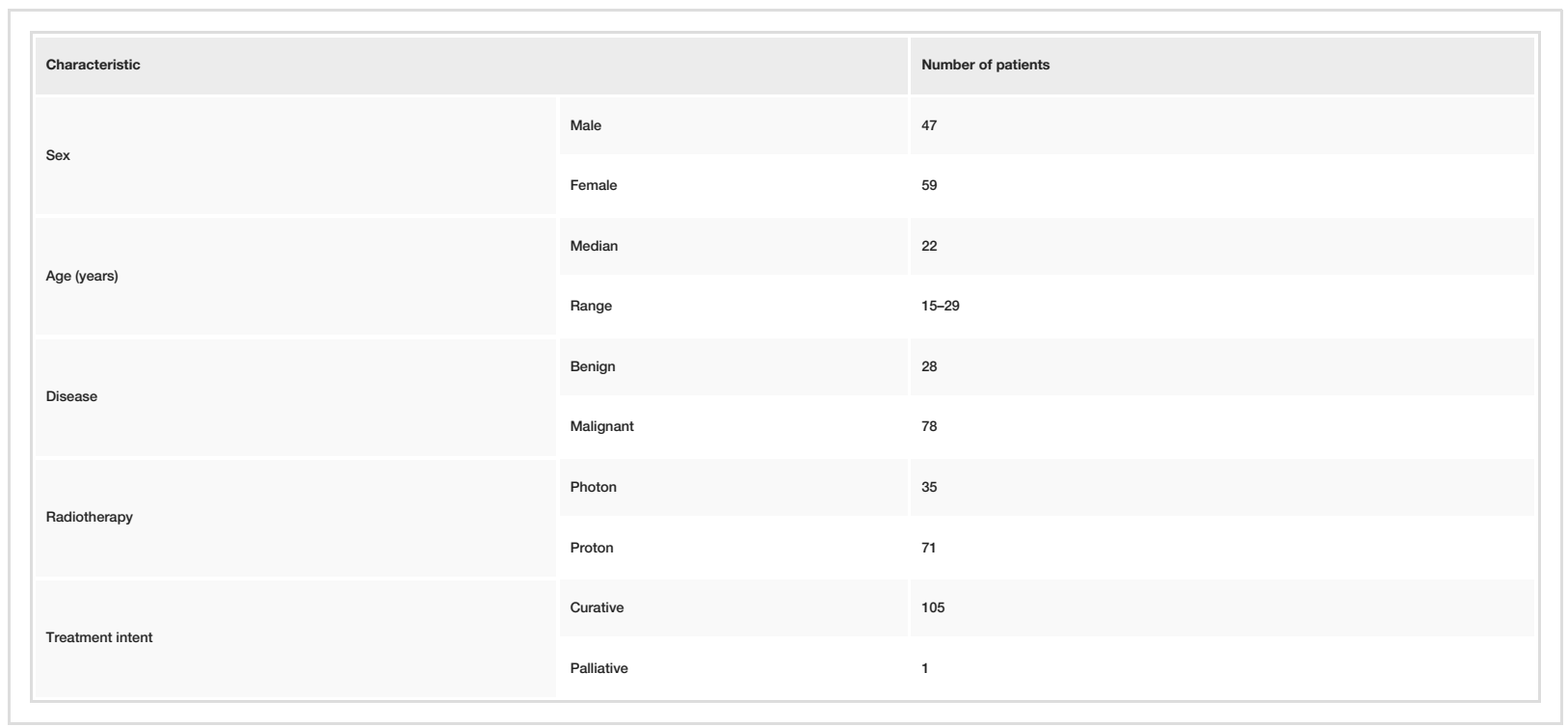

TABLE 1: Patient characteristics.

Overall, 35 and 71 patients received photon and proton therapy, respectively. Photon therapy was performed from 2004 to 2015 for 70 patients, and 35 of 70 patients were followed-up for less than two years (28 died due to cancer and seven were lost to follow-up). Proton therapy was performed from 1986 to 2017 for 100 patients. Of the 100 patients, 29 were followed-up for less than two years (17 died due to cancer and 12 were lost to follow-up). The median age of the patients was 22 years, ranging from 15 to 29 years. Of the 106 patients, 47 were male and 59 were female. All but one patient received radiotherapy with a curative intent. The only palliative treatment was photon radiotherapy with 34.4 Gy given in 23 fractions for central nervous system germinoma. The target disease was benign and malignant in 29 and 77 patients, respectively. Benign disease included arteriovenous malformation $(n=23)$, pituitary tumor $(n=3)$, meningioma $(n=1)$, schwannoma $(n=1)$, and thymoma $(n=1)$. Various malignant diseases were treated, especially with proton beam therapy (PBT). Photon radiotherapy mainly treated hematologic malignancies and cervical cancer. The details of the diseases are shown in Tables 2 and 3. 


\section{Cureus}

\begin{tabular}{|c|c|c|c|}
\hline Disease & Total $(n=106)$ & Proton therapy $(n=71)$ & Photon therapy $(\mathrm{n}=35)$ \\
\hline ALL & 4 & 0 & 4 \\
\hline AML & 2 & 0 & 2 \\
\hline AVM & 23 & ${ }^{23}$ & 0 \\
\hline Brain tumor & 20 & 14 & 6 \\
\hline Breast cancer & 4 & 0 & 4 \\
\hline Cervical cancer & 5 & 0 & 5 \\
\hline Chordoma & 5 & 5 & 0 \\
\hline Chondrosarcoma & 1 & 1 & 0 \\
\hline HCC & 3 & 3 & 0 \\
\hline Hepatoblastoma & 1 & 1 & 0 \\
\hline Head and neck cancer & 5 & 2 & 3 \\
\hline Hodgkin's lymphoma & 5 & 3 & 2 \\
\hline Non-Hodgkin's Iymphoma & 8 & 2 & 6 \\
\hline Neuroblastoma & 2 & 2 & 0 \\
\hline Osteosarcoma & 2 & 2 & 0 \\
\hline Rhabdomyosarcoma & 4 & 4 & 0 \\
\hline PNET & 3 & 1 & 2 \\
\hline Testicular tumor & 2 & 1 & 1 \\
\hline Others & 7 & 7 & 0 \\
\hline
\end{tabular}

\section{TABLE 2: Details of the diseases.}

ALL: acute lymphoblastic leukemia; AML: acute myelogenous (myeloid) leukemia; AVM: arteriovenous malformation; HCC: hepatocellular carcinoma; PNET: primitive neuroectodermal tumor

\begin{tabular}{|c|c|c|c|}
\hline Radiotherapy site & Total $(n=106)$ & Proton therapy $(n=71)$ & Photon therapy $(n=35)$ \\
\hline Brain & 43 & 38 & 5 \\
\hline Skull base & 6 & 6 & 0 \\
\hline Head and neck & 12 & 7 & 5 \\
\hline Chest & 18 & 10 & 8 \\
\hline Abdomen/pelvis & 15 & 9 & 6 \\
\hline Extremities & 1 & 1 & 0 \\
\hline Spine & 1 & 0 & 1 \\
\hline TBI & 9 & 0 & 9 \\
\hline CSI & 1 & 0 & 1 \\
\hline
\end{tabular}

TABLE 3: Radiotherapy sites.

TBI: total body irradiation: CSI: cerebrospinal irradiation 


\section{Cureus}

The primary endpoint of this study was late toxicities, and the secondary endpoint was life events, including marriage, giving birth, and employment or education status. Toxicities were graded according to the Common Terminology Criteria for Adverse Events version 4.0 [5].

\section{Results}

The median follow-up period in all patients was 62 months (range: 24.0-293.6 months) and 68 months for survivors. Late toxicities were observed in 45 patients. Details of the toxicities are shown in Table 4 . Grade 3 or higher toxicity was observed in 20 patients. Grade 3 toxicity was seen in 17 patients, of which endocrine disorders occurred in eight patients who subsequently received hormonal replacement therapy. Other cases of grade 3 toxicity included gastrointestinal ulcer $(n=1)$, skin ulcer $(n=1)$, brain necrosis $(n=1)$, brainstem infarction $(n=1)$, low vision $(n=2)$, cataract $(n=2)$, and ileus $(n=1)$. Grade 4 toxicity was observed in two patients. A patient with moyamoya disease received total body irradiation at a dose of $12 \mathrm{~Gy}$ in four fractions for nasal natural killer (NK)/T-cell lymphoma when she was 29 years old. Facial bone deformity occurred in a patient who received photon therapy for nasal NK/T-cell lymphoma at a dose of 50 Gy in 25 fractions when he was 22 years old. Grade 5 toxicity in the form of myelodysplastic syndrome occurred in a patient who received proton therapy for Ewing sarcoma (local irradiation of 55.8 Gy (RBE) in 31 fractions) when she was 15 years old. Secondary cancer was not observed outside of the one case of myelodysplastic syndrome. Depression was observed in two patients.

\begin{tabular}{|c|c|c|c|c|c|}
\hline Grade & 1 & 2 & 3 & 4 & 5 \\
\hline Skin atrophy/ulcer & 1 & 3 & 1 & 0 & 0 \\
\hline Facial deformation & 0 & 0 & 0 & 1 & 0 \\
\hline Cataract/low vision & 0 & 0 & 4 & 0 & 0 \\
\hline Ear (noise, hearing disorder, otitis) & 1 & 3 & 0 & 0 & 0 \\
\hline Dry mouth & 2 & 0 & 0 & 0 & 0 \\
\hline Hoarse voice & 1 & 0 & 0 & 0 & 0 \\
\hline Seizures & 0 & 5 & 0 & 0 & 0 \\
\hline Headache & 1 & 3 & 0 & 0 & 0 \\
\hline Brain atrophy & 1 & 0 & 0 & 0 & 0 \\
\hline Brain necrosis & 0 & 0 & 1 & 0 & 0 \\
\hline CVD (moyamoya disease, infarction) & 0 & 0 & 1 & 1 & 0 \\
\hline Endocrine disorder & 0 & 0 & 8 & 0 & 0 \\
\hline Pneumonia & 1 & 0 & 0 & 0 & 0 \\
\hline Enteritis/gastrointestinal perforation & 0 & 1 & 1 & 0 & 0 \\
\hline Ileus & 0 & 0 & 1 & 0 & 0 \\
\hline Extremity pain & 0 & 1 & 0 & 0 & 0 \\
\hline Depression & 0 & 2 & 0 & 0 & 0 \\
\hline Lymphedema & 2 & 0 & 0 & 0 & 0 \\
\hline Myelodysplastic syndrome & 0 & 0 & 0 & 0 & 1 \\
\hline Total & 10 & 18 & 17 & 2 & 1 \\
\hline
\end{tabular}

TABLE 4: Toxicities observed in patients.

CVD: cardiovascular disease

Regarding life events, 15 and 88 patients were married and unmarried at the start of radiotherapy, respectively, and three had unknown marital status. Of the 88 unmarried patients, five were married after radiotherapy, and 83 were unmarried at the final follow-up. 
A total of 90 patients did not have a child at the start of radiotherapy. Of the 90 patients, 45 were female, and five gave birth after radiotherapy. No male patients had children at the start of the treatment.

Occupation and education were evaluated in 71 patients who received PBT. Of the 71 patients, 33 were students, 21 were employed, and 16 were unemployed at the start of radiotherapy. Occupational information was unknown for the remaining two patients. Of the 33 student patients, eight (24\%) were employed, 11 (33\%) were at a higher grade of education at the last follow-up, and 14 had unknown educational status. Of the 21 employed patients, 17 (81\%) were still in the same jobs at the last follow-up, but four had lost their jobs. Of the 16 unemployed patients, all were still unemployed at the final follow-up (Figure 1).

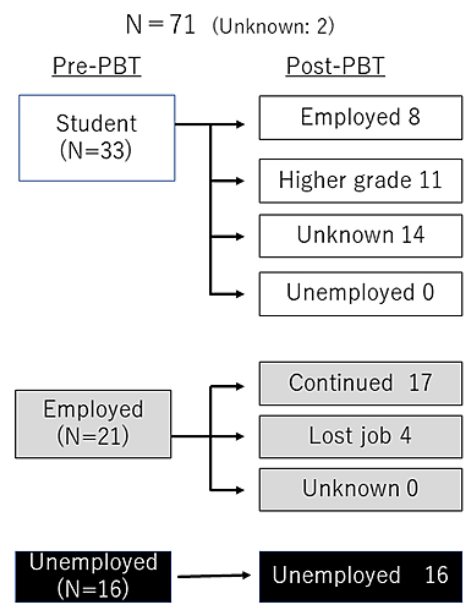

FIGURE 1: Employment and education status of 71 patients.

\section{Discussion}

In Japan, cancer morbidity is increasing in those over 45 years old [6] and cancer mortality rate is increasing in those over 55 years old [7]. Hence, AYAs are rare among patients with common cancers. Furthermore, life stages rapidly change in the AYA generation and differ from those in other generations. Adolescents are in a stage of mental and social development and have not started working yet or have not become economically independent. Young adults start to become independent and start working. Therefore, the treatment effects of the same radiotherapy regimen may differ based on their circumstances. Naturally, AYA cancer survivors experience cancer-related distress [8-13], such as fear of cancer recurrence. Additionally, many factors influencing this distress have been suggested, including multimodal treatment, history of psychological distress [10], employment, type of cancer [9], age [11], and gender [8]. Wang et al. evaluated cancer-related worry in 15-26-year olds using the six-item Cancer Worry Scale [8]. They concluded that female survivors and higher treatment intensity were associated with increased cancer worry regarding infertility and/or secondary cancer. Mattoson et al. also reported that $85 \%$ of the patients, suffering from gynecological cancer at 19-39 years, were suffering from cancer-related distress, including fear of cancer recurrence (61\%), anxiety (53\%), depression (51\%), fear of death (32\%), concerns regarding sexuality (34\%), fertility (27\%), and change in body image (27\%) [10]. Shay et al. showed that the rate of fear of recurrence was significantly higher among AYA survivors than among those diagnosed at an older age [9]. Beattie et al. also showed that younger age was associated with higher fear of recurrence among breast cancer patients [11]. In our hospital, we performed follow-up for pediatric patients with a radiation oncologist, pediatrician, and/or surgeon to respond to various changes. Similar to dealing with pediatric patients, follow-up by multiple experts (radiation oncologist, clinical psychologist, social worker, gynecologist, etc.) may be needed for AYA cancer survivors to reduce cancer-related events.

The Children's Oncology Group recommends that patients receiving radiotherapy to the head and neck undergo dental examination and cleaning every six months because of the risk of dental decay [14]. Hamilton et al. reported late effects in AYA head and neck cancer survivors who survived for more than five years after diagnosis. In this study, $78 \%$ of the survivors had at least one late toxicity from radiotherapy, including chronic skin changes, osteonecrosis, and hearing loss. Additionally, a second malignancy was observed in $17 \%$ of the patients. Lim et al. reported the results of treatment of 176 AYAs with brain and skull base tumors at the Paul Scherrer Institute [15]. PBT is expected to have a lower rate of toxicities due to its favorable dose distribution, and severe (greater than grade 3) ototoxicity and neurotoxicity were observed in $3.4 \%$ and $2.9 \%$ of the patients, respectively. However, the rate of unemployment was $9.5 \%$ pre-PBT, increasing up to $23.8 \%$ post-PBT. Adverse events caused by PBT and having to take time for surgery and PBT affected employment. 
In this study, $76 \%$ of the patients who were students could find jobs or continued higher-grade education, and $81 \%$ of the patients who had a job continued their previous jobs. However, none of the patients who were unemployed at the time of radiotherapy could find a job after radiotherapy. This result may be due to the lifetime employment system in Japan, and suggests that once a patient loses their job, it is difficult for them to find new jobs. In addition, the ratio of number of marriages to women giving birth was also low. Regarding birth or marriage, Gerstl et al. conducted a meta-analysis of reproduction among AYA female cancer survivors. They suggested a low rate of live births for patients who received chemotherapy or radiotherapy compared to surgery alone: $10 \%$ among patients who received chemotherapy alone, $18 \%$ among patients who received radiotherapy alone, and $44 \%$ among patients who received surgery alone. They also suggested that among AYA patients who became pregnant, $79 \%$ gave birth, and $22 \%$ of these births were preterm [16]. In our study, marriage and birth rates were low. At the last follow-up, 83 of the 88 patients were unmarried and five of the 45 female patients gave birth after radiotherapy. Some studies have shown that PBT probably reduces the risk of late toxicity among younger patients [17-19]. However, we did not compare patients who received PBT and photon radiotherapy because the disease and tumor location were very different among patients treated with these two modalities, and no secondary malignancies were observed in this study, possibly due to the short follow-up period.

The main limitations of this study are its retrospective design and the short follow-up period.

\section{Conclusions}

This is one of the largest studies focusing on life after radiation therapy, suggesting that cancer treatment may impact life events. More detailed data collected over a longer period of time are necessary to clarify the effect of radiotherapy on the future lives for AYAs.

\section{Additional Information}

\section{Disclosures}

Human subjects: Consent was obtained or waived by all participants in this study. Tsukuba Clinical Research \& Development Organization issued approval R01-144. Animal subjects: All authors have confirmed that this study did not involve animal subjects or tissue. Conflicts of interest: In compliance with the ICMJE uniform disclosure form, all authors declare the following: Payment/services info: All authors have declared that no financial support was received from any organization for the submitted work. Financial relationships: All authors have declared that they have no financial relationships at present or within the previous three years with any organizations that might have an interest in the submitted work. Other relationships: All authors have declared that there are no other relationships or activities that could appear to have influenced the submitted work.

\section{References}

1. Thewes B, Kaal SEJ, Custers JAE, et al.: Prevalence and correlates of high fear of cancer recurrence in late adolescents and young adults consulting a specialist adolescent and young adult (AYA) cancer service. Support Care Cancer. 2018, 26:1479-87. 10.1007/s00520-017-3975-2

2. Henderson TO, Parsons SK, Wroblewski KE, et al.: Outcomes in adolescents and young adults with Hodgkin lymphoma treated on US cooperative group protocols: an adult intergroup (E2496) and Children's Oncology Group (COG AHOD0031) comparative analysis. Cancer. 2018, 124:136-44. 10.1002/cncr.30979

3. Bleyer A, Barr R, Hayes-Lattin B, Thomas D, Ellis C, Anderson B: The distinctive biology of cancer in adolescents and young adults. Nat Rev Cancer. 2008, 8:288-98. 10.1038/nrc2349

4. National Cancer Institute: cancer epidemiology in older adolescents and young adults 15 to 29 Years of Age - including SEER incidence and survival: 1975-2000. (2006). Accessed: April 14, 2021: https://seer.cancer.gov/archive/publications/aya/.

5. Common Terminology Criteria for Adverse Events v4.0 . (2010). Accessed: April 14, 2021: https://ctep.cancer.gov/protocoldevelopment/electronic_applications/ctc.htm\#ctc_40.

6. Cancer Registry and Statistics. Cancer Information Service NCC, Japan (Monitoring of Cancer Incidence in Japan (MCIJ). (2020). Accessed: April 14, 2021: http://ganjoho.jp/reg_stat/statistics/dl/index.html\#incidence4pref.

7. Cancer Registry and Statistics. Cancer Information Service NCC, Japan (Vital Statistics of Japan) . (2021). Accessed: April 14, 2021: https://www.ncc.go.jp/en/index.html.

8. Wang R, Syed IA, Nathan PC, Barr RD, Rosenberg-Yunger ZR, Klassen AF: Exploring cancer worry in adolescent and young adult survivors of childhood cancers. J Adolesc Young Adult Oncol. 2015, 4:192-9. 10.1089/jayao.2015.0029

9. Shay LA, Carpentier MY, Vernon SW: Prevalence and correlates of fear of recurrence among adolescent and young adult versus older adult post-treatment cancer survivors. Support Care Cancer. 2016, 24:4689-96. 10.1007/s00520-016-3317-9

10. Mattsson E, Einhorn K, Ljungman L, Sundström-Poromaa I, Stålberg K, Wikman A: Women treated for gynaecological cancer during young adulthood - a mixed-methods study of perceived psychological distress and experiences of support from health care following end-of-treatment. Gynecol Oncol. 2018, 149:464-9. 10.1016/j.ygyno.2018.03.055

11. Lebel S, Beattie S, Arès I, Bielajew C: Young and worried: age and fear of recurrence in breast cancer survivors. Health Psychol. 2013, 32:695-70. 10.1037/a0030186

12. Yang Y, Li W, Wen Y, et al.: Fear of cancer recurrence in adolescent and young adult cancer survivors: a 


\section{Cureus}

systematic review of the literature. Psychooncology. 2019, 28:675-86. 10.1002/pon.5013

13. Hamilton SN, Arshad O, Kwok J, Tran E, Fuchsia Howard A, Serrano I, Goddard K: Documentation and incidence of late effects and screening recommendations for adolescent and young adult head and neck cancer survivors treated with radiotherapy. Support Care Cancer. 2019, 27:2609-16. 10.1007/s00520-0184559-5

14. Long-term follow-up guidelines for survivors of childhood, adolescents, and young adult cancers Version 5.0 . (2018). Accessed: April 14, 2021: http://www.survivorshipguidelines.org/pdf/2018/COG_LTFU_Guidelines_v5.pdf.

15. Lim PS, Tran S, Kroeze SGC, et al.: Outcomes of adolescents and young adults treated for brain and skull base tumors with pencil beam scanning proton therapy. Pediatr Blood Cancer. 2020, 67:28664 10.1002/pbc. 28664

16. Gerstl B, Sullivan E, Chong S, Chia D, Wand H, Anazodo A: Reproductive outcomes after a childhood and adolescent young adult cancer diagnosis in female cancer survivors: a systematic review and meta-analysis. J Adolesc Young Adult Oncol. 2018, 7:6. 10.1089/jayao.2018.0036

17. Mizumoto M, Murayama S, Akimoto T, et al.: Proton beam therapy for pediatric malignancies: a retrospective observational multicenter study in Japan. Cancer Med. 2016, 5:1519-25. 10.1002/cam4.743

18. Mizumoto M, Murayama S, Akimoto T, et al.: Long-term follow-up after proton beam therapy for pediatric tumors: a Japanese national survey. Cancer Sci. 2017, 108:444-7. 10.1111/cas.13140

19. Mizumoto M, Oshiro Y, Yamamoto T, Kohzuki H, Sakurai H: Proton beam therapy for pediatric brain tumor . Neurol Med Chir (Tokyo). 2017, 57:343-55. 10.2176/nmc.ra.2017-0003 